\title{
Cosmic Ray Diffusion in the W44 Region with the MAGIC Telescopes
}

\author{
On behalf of the MAGIC Collaboration: \\ Sara Rebecca Gozzini \\ Deutsches Elektronen-Synchrotron (DESY), D-15738 Zeuthen, Germany \\ E-mail: sara.rebecca.gozzini@gmail.com

\section{Yoshitaka Hanabata} \\ Institute for Cosmic-Ray Research, University of Tokyo, 5-1-5 Kashiwanoha, Kashiwa, Chiba \\ 277-8582, Japan \\ E-mail: hanabata@icrr.u-tokyo.ac.jp
}

\section{Pierre Colin*}

Max-Planck-Institut für Physik, D-80805 München, Germany

E-mail: colin@mppmu.mpg.de

\section{Roberta Zanin ${ }^{\dagger}$}

Universitat de Barcelona (ICC, IEEC-UB), E-08028 Barcelona, Spain

E-mail: rzaninaam.ub.es

\section{Giovanna Pedaletti}

Deutsches Elektronen-Synchrotron (DESY), D-15738 Zeuthen, Germany

E-mail: giovanna.pedaletti@desy.de

\section{Emma de Oña Wilhelmi}

Institut de Ciències de l'Espai (IEEC-CSIC), E-08193 Bellaterra, Spain

E-mail: wilhelmidice.cat

\section{Diego F. Torres}

Institut de Ciències de l'Espai (IEEC-CSIC), E-08193 Bellaterra, Spain

E-mail: dtorresdieec.uab.es

\section{Julian Krause}

Max-Planck-Institut für Physik, D-80805 München, Germany

E-mail: jkrause@mpp.mpg.de

For the NANTEN Collaboration:

\section{Yasuo Fukui}

Department of Physics, Nagoya University, Furo-cho, Chikusa-ku, Nagoya 464-8601, Japan E-mail: Eukui@a.phys.nagoya-u.ac.jp

\section{Satoshi Yoshiike}

Department of Physics, Nagoya University, Furo-cho, Chikusa-ku, Nagoya 464-8601, Japan E-mail: yoshiike@a.phys.nagoya-u.ac.jp 


\section{Hidetoshi Sano}

Institute for Advanced Research, Nagoya University, Furo-cho, Chikusa-ku, Nagoya 464-8601, Japan

E-mail: sano@a.phys.nagoya-u.ac.jp

\section{On behalf of the FERMI-LAT Collaboration:}

\section{Francesco Giordano}

Dip. Interateneo di Fisica "M. Merlin" Via Amendola 17370126 Bari

E-mail: Erancesco.giordano@ba.infn.it

\section{Leonardo Di Venere}

Dip. Interateneo di Fisica "M. Merlin" Via Amendola 17370126 Bari

E-mail: Leonardo,divenereaba.infn.it

The W44 region includes the supernova remnant SNR G34.7-0.4 and two additional surrounding GeV sources, revealed with Fermi Large Area Telescope (LAT); the whole system is embedded in the giant molecular cloud G34.8-0.6. In the hypothesis that hadrons are accelerated at the SNR shock, the geometry of the system suggests a possible signature of their diffusion and interaction with the cloud away from the acceleration site, resulting in $\gamma$-ray emission offset from the radio SNR shell. In order to test the diffusion scenario, the GeV source with the harder spectrum (W44 SRC1) has been observed in very-high energy $\gamma$ rays with the MAGIC Telescopes. No significant very-high energy signal has been found from W44 SRC1 in about 100 hours of data. Upper limits on the integral flux of this region have been computed at energies above $50 \mathrm{GeV}$. The source extension will be derived by the GeV emission provided by Fermi-LAT. The surrounding molecular cloud has been studied based on HI and CO maps provided by the NANTEN collaboration. We used these data to constrain the cosmic ray diffusion coefficient in the region.

The 34th International Cosmic Ray Conference,

30 July- 6 August, 2015

The Hague, The Netherlands

* Speaker.

$\dagger$ Speaker. 


\section{Introduction}

Supernova remnants (shortened as SNR) are candidate acceleration sites for those protons and hadrons that are detected as cosmic rays. Of particular interest are those Galactic SNR in interaction with nearby molecular clouds. In these environments, hadrons accelerated at the SNR shock scatter onto surrounding target gas. Neutral mesons $\left(\pi^{0}, \eta^{0}\right)$, produced in these hadronic interactions, decay originating the $\gamma$-ray emission. For this reason, the presence of a molecular cloud enhances the resulting $\gamma$-ray flux. In these systems, moreover, a correlation is expected between the position of the $\gamma$-ray source and the location of the molecular material.

At high energies (defined here as $\mathrm{HE}, 30 \mathrm{MeV} \lesssim E \lesssim 100 \mathrm{GeV}$ ), evidence of hadronic acceleration has been found in two systems consisting of a middle-age SNR interacting with molecular cloud, (W44 and IC443, see Ackermann et al. 2013). For both objects, not only $\gamma$-ray emission has been detected in correspondence with the surrounding molecular cloud, but it has also been possible to identify the signature of the $\pi^{0}$ decay cross section. Other cases of $\gamma$-ray emission correspondent with molecular clouds have been found (e.g. W28, Aharonian et al. 2008).

The reaction yielding gamma rays, $p N \rightarrow \pi^{0} X \rightarrow 2 \gamma+X$, could happen either in the direct vicinity of the front, or after the cosmic ray particles diffuse for a certain distance away from their acceleration site into the surrounding medium (Hanabata et al. 2014; Torres et al. 2010; Uchiyama et al. 2012). The dynamics of the escape and diffusion of cosmic rays from their accelerators can be modelled with analytical solutions to the diffusion-loss equations (Aharonian \& Atoyan 1996; Ohira et al. 2011). These, however, require a parametrisation of the diffusion coefficient, and some assumptions on the characteristic of the accelerator. The diffusion process is energy dependent, and can therefore alter the spectrum of the injected accelerated particles, with the higher energy particles diffusing faster. The spectral change is more evident the larger the separation between the accelerator site and the target material.

Here we report on the observations of the W44 region, that consists of the W44 supernova remnant (G34.7-0.4), embedded in the giant molecular cloud G34.8-0.6, and of the two diametrically opposed GeV sources W44 SRC1 and W44 SRC2, revealed with Fermi-LAT when subtracting W44 as a background (Uchiyama et al. 2012).

W44 is a close-by, middle-aged SNR in its radiative phase; its distance is estimated as 2.9 $\mathrm{kpc}$; dense material is present all around in the giant molecular cloud G34.8-0.6, offering target for hadronic interactions.

Bright compact filaments, seen in X-rays and originated by non-thermal synchrotron radiation, are a tracer of magnetic field amplification, crucial condition for an accelerator to reach energies above $O(10) \mathrm{GeV}$. The W44 region has previously been observed with Agile and Fermi in the GeV range, and it has been recently re-analysed Cardillo et al. (2014); its spectral energy distribution shows a neat hadronic signature, having a clear-cut rise in correspondence of half the mass of the lightest neutral meson.

Fermi measured a spectral index of $\alpha \sim 3$ for the W44 SNR. This value is larger than the prediction from standard diffuse shock acceleration. Given the steep spectrum and the age of the SNR, this suggests escape of high-energy particles away from the SNR into the surrounding medium. This interpretation is supported by the detection of W44 SRC1 and W44 SRC2 in HE (Uchiyama et al. 2012). 
The picture of the diffusion away from the accelerator is complete with observations in veryhigh energies (defined as VHE, $50 \mathrm{GeV} \lesssim E \lesssim 50 \mathrm{TeV}$ ). Due to the limited field of view of the MAGIC Telescopes, we had to choose one of the two GeV sources. In this work, we focus on W44 SRC1, the North-West excess, which has the highest flux and the flatter spectrum.

\section{References}

Ackermann, M., Ajello, M., Allafort, A. et al. 2013, Science, 339, 807A

Aharonian \& Atoyan 1996 ApJ, 309, 917

Aharonian, F., Akhperjanian, A. G., Bazer-Bachi, A. R et al. 2008, A\&A, 481, 401A

Cardillo, M., Tavani, M., Giuliani, A. et al. 2014, A\&A, 565A, 74C

Hanabata, Y., Katagiri, H., Hewit, J. W., et al. 2014, ApJ, 786, 145

Ohira, Y., Murase, K. \& Yamazaki, R. 2011 MNRAS,410,1577O

Torres D. F. et al. 2010, MNRAS, 408, 1257

Uchiyama, Y. et al. 2012, ApJ, 749L, 35U

Yoshiike, S., Fukuda, T., Sano, H. et al. 2013, ApJ, 768, 179Y 